\title{
Colloidal gold-loaded, biodegradable, polymer-based stavudine nanoparticle uptake by macrophages: an in vitro study
}

This article was published in the following Dove Press journal:

International Journal of Nanomedicine

12 December 2012

Number of times this article has been viewed

\section{Sumit Basu',2 \\ Biswajit Mukherjee' \\ Samrat Roy Chowdhury' \\ Paramita Paul' \\ Rupak Choudhury ${ }^{3}$ \\ Ajeet Kumar' \\ Laboni Mondal' \\ Chowdhury Mobaswar \\ Hossain' \\ Ruma Maji \\ 'Department of Pharmaceutical Technology, Jadavpur University, Kolkata, India; ${ }^{2}$ Department of Pharmacological and Pharmaceutical Sciences, College of Pharmacy, University of Houston, Houston, TX, USA; ${ }^{3}$ Department of Biochemistry, Ballygunge Science College, Kolkata, India \\ Video abstract

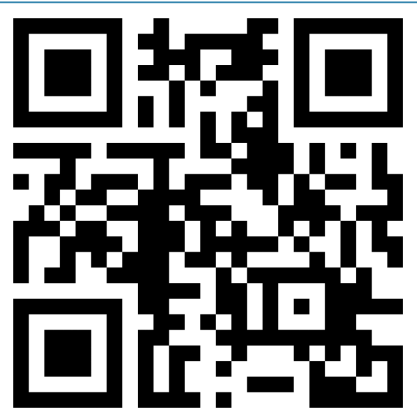 \\ Point your SmartPhone at the code above. If you have a QR code reader the video abstract will appear. Or use: http://dvpr.es/UdGa27}

Correspondence: Biswajit Mukherjee

Department of Pharmaceutical

Technology, Jadavpur University,

Kolkata 700032, India

Tel/fax +913324146677

Email biswajit55@yahoo.com
Objective: We describe the development, evaluation, and comparison of colloidal gold-loaded, poly(d,l-lactic-co-glycolic acid)-based nanoparticles containing anti-acquired immunodeficiency syndrome drug stavudine and uptake of these nanoparticles by macrophages in vitro.

Methods: We used the following methods in this study: drug-excipient interaction by Fourier transform infrared spectroscopy, morphology of nanoparticles by field-emission scanning electron microscopy, particle size by a particle size analyzer, and zeta potential and polydispersity index by a zetasizer. Drug loading and in vitro release were evaluated for formulations. The best formulation was incorporated with fluorescein isothiocyanate. Macrophage uptake of fluorescein isothiocyanate nanoparticles was studied in vitro.

Results: Variations in process parameters, such as speed of homogenization and amount of excipients, affected drug loading and the polydispersity index. We found that the drug was released for a prolonged period (over 63 days) from the nanoparticles, and observed cellular uptake of stavudine nanoparticles by macrophages.

Conclusion: Experimental nanoparticles represent an interesting carrier system for the transport of stavudine to macrophages, providing reduced required drug dose and improved drug delivery to macrophages over an extended period. The presence of colloidal gold in the particles decreased the drug content and resulted in comparatively faster drug release.

Keywords: stavudine, poly(d,l-lactic-co-glycolic acid), nanoparticles, colloidal gold, uptake by macrophages

\section{Introduction}

Optimization of the pharmacological action of a drug along with reduction in its toxic side effects is a prime prerequisite for an ideal drug-delivery system. Colloidal drug carriers can provide site-specific or targeted drug delivery along with optimal drug release. Among these carriers, nanoparticles and liposomes have been widely investigated. Due to various technical problems, such as poor stability and low entrapment efficiency of liposomes, polymeric nanoparticles were proposed as a suitable alternative. One of the most attractive areas of research using polymeric nanoparticles is the controlled delivery of drug following parenteral, oral, pulmonary, nasal, and topical routes of administration. Polymeric nanoparticles can also be targeted to specific cells and tissues in the body by virtue of their small size and by functionalizing their surface with polymers and appropriate ligands. ${ }^{2}$ Further, polymeric nanoparticles usually overcome stability issues of liposomes and can minimize the therapeutic dose and thus minimize drug-induced side effects by sustained drug release. ${ }^{3}$ A diverse range of materials has been used as drug carriers, including polymers ${ }^{4}$ and dendrimers, ${ }^{5}$ and nanomaterials 
such as nanotubes, ${ }^{6}$ nanorods, ${ }^{7}$ and nanoparticles. ${ }^{8}$ Gold nanoparticles provide promising scaffolds for drug and gene delivery. Their unique features, such as tunable core size, monodispersity, large surface-to-volume ratio, and easy functionalization with virtually any molecule or biomolecule enable their effective targeting, transport, and tuning of delivery processes. ${ }^{9}$ Gold nanoparticles are the preferred delivery system because of their relatively lesser intrinsic toxicity toward the normal cell. ${ }^{9}$

Nanoparticles consisting of gold offer enhanced absorption and scattering, good biocompatibility, facile synthesis, ${ }^{4}$ and conjugation to a variety of biomolecular ligands, antibodies, and other targeting moieties, ${ }^{5}$ making them suitable for use in biochemical sensing and detection, ${ }^{10-12}$ medical diagnostics, and therapeutic applications. ${ }^{13,14}$

Acquired immunodeficiency syndrome (AIDS) is a disease of the human immune system caused by the human immunodeficiency virus (HIV). ${ }^{4}$ This condition progressively reduces the effectiveness of the immune system and leaves the individual susceptible to opportunistic infection and tumor. It is transmitted through direct contact of a mucous membrane or the bloodstream with a bodily fluid containing HIV, such as blood, semen, vaginal fluid, pre-seminal fluid, and breast milk. ${ }^{5}$ There is currently no vaccine or cure for HIV or AIDS. The only known methods of prevention are based on avoiding exposure to the virus and antiretroviral treatment once affected. The antiviral therapy has unpleasant side effects, including peripheral neuropathy, acute pancreatitis, abdominal pain, diarrhea, malaise, nausea, and fatigue. AIDS patients are generally treated with nucleoside or nucleotide reverse transcriptase inhibitors that inhibit reverse transcription by blocking the reverse transcriptase enzyme responsible for conversion from single-stranded RNA to double-stranded DNA in HIV. Zidovudine, didanosine, zalcitabine, stavudine, lamivudine, and abacavir are nucleoside analogs and tenofovir and adefovir are nucleotide analogs used as reverse transcriptase inhibitors for HIV infection. These drugs have severe side effects at higher dose.

In the present study we used stavudine, which has a short half-life and poor bioavailability. Nanoparticles are used as a drug carrier for delivery of a drug to overcome the problems of short half-life, poor bioavailability, and strong side effects. In this study we developed, evaluated, and compared a poly(d,l-lactic-co-glycolic acid) (PLGA)based nanoparticulate drug delivery system, with or without gold nanoparticles, containing stavudine. We also studied the uptake of nanoparticles by macrophages in vitro, since HIV accumulates in macrophages during the early phase (first 1 to 2 years) of infection. ${ }^{6}$

In the present study, PLGA was used, since it is an FDAapproved biodegradable polymer capable of drug release in a sustained manner. In addition to the drug, gold nanoparticles were incorporated into the polymeric nanoparticle matrices with the following expectations: first, since a gold nanoparticle has a higher surface-to-volume ratio and gold can hold many different molecules attached physically on its surface, we wanted to see whether gold can help enhance stavudine-loading in the nanoparticle; second, gold nanoparticles and their conjugates have been reported to inhibit HIV transinfection; ${ }^{15}$ third, the presence of gold in PLGA nanoparticles can be used for imaging to localize the presence of nanoparticles in organs and tissues in vivo, which is the future program in the study.

\section{Materials and methods Materials}

Stavudine was obtained from Cipla Ltd (gift sample) (Baddi, India). PLGA (85:15; molecular weight [MW] 50,000-75,000), fluorescein isothiocyanate (FITC), chloroauric acid $\left(\mathrm{HAuCl}_{4}\right)$, and trisodium citrate were purchased from Sigma-Aldrich Corporation (Bangalore, India). Polyvinyl alcohol ([PVA] MW 30,000-70,000) was obtained from SD Fine Chem Ltd (Mumbai, India). Dichloromethane (DCM) was purchased from E Merck (India) Ltd (Mumbai, India). Disodium hydrogen orthophosphate and potassium hydrogen phosphate were obtained from Process Chemical Industries (Kolkata, India). All other chemicals used were of analytic grade.

\section{Preparation of polymeric drug-loaded nanoparticles}

PVA solutions were prepared in $1.5 \%$ (1.5 g PVA in $100 \mathrm{~mL}$ water) and $2.5 \%$ ( $0.25 \mathrm{~g}$ of PVA in $10 \mathrm{~mL}$ water $)$ concentrations. We then dissolved $250 \mathrm{mg}$ PLGA in $2 \mathrm{~mL}$ DCM. The total amount of stavudine (Table 1) was dissolved in $0.5 \mathrm{~mL}$ of $2.5 \%$ PVA. The drug solution $(0.5 \mathrm{~mL})$ was then added drop-wise into $2 \mathrm{~mL}$ of the PLGA solution while homogenizing, and the mixture was homogenized (WiseTis homogenizer; Daihan Scientific Co Ltd, Seoul, South Korea) at different speeds for different formulations (Table 1) for 4 minutes. This resulted in a water-in-oil (w/o) type of emulsion. The emulsion was gradually added to $75 \mathrm{~mL}$ of $1.5 \%$ PVA solution and homogenized at the same speed according to the formulation for 6 minutes, which produced a water-in-oil-in-water (w/o/w) type of emulsion. The emulsion 
Table I Composition of experimental nanoparticles with their drug loading and entrapment efficiency

\begin{tabular}{|c|c|c|c|c|c|c|}
\hline $\begin{array}{l}\text { Formulation } \\
\text { code }\end{array}$ & $\begin{array}{l}\text { Stavudine:PLGA } \\
\text { (mg:mg) }\end{array}$ & $\begin{array}{l}\text { Presence of } \\
\text { colloidal gold }\end{array}$ & $\begin{array}{l}\text { Speed of } \\
\text { homogenization (rpm) }\end{array}$ & $\begin{array}{l}\text { Amount } \\
\text { recovered }^{\text {a }}(\mathrm{mg})\end{array}$ & $\begin{array}{l}\text { Drug } \\
\text { loading (\%) }\end{array}$ & $\begin{array}{l}\text { Entrapment } \\
\text { efficiency }(\%)\end{array}$ \\
\hline $\mathrm{SI}$ & $20: 250$ & No & 15,000 & 165.4 & 5.69 & 76.83 \\
\hline S2 & $20: 250$ & Yes & 15,000 & 187.2 & 5.08 & 68.57 \\
\hline S3 & $25: 250$ & No & 16,000 & 150 & 7.93 & 87.23 \\
\hline S4 & $25: 250$ & Yes & 16,000 & 186.6 & 6.15 & 67.63 \\
\hline S5 & $0: 250$ & No & 15,000 & 152 & NA & NA \\
\hline
\end{tabular}

Notes: aRecovery $(\mathrm{mg})=$ amount of lyophilized formulation obtained $(\mathrm{mg})$ out of total amount of drug and excipients used (mg); ${ }^{\mathrm{b}}$ Drug loading (actual) $(\%)=$ Amount of drug in nanoparticles $\times 100 /$ Amount of nanoparticles obtained; 'Drug entrapment efficiency (\%) = Drug loading (actual) $(\%) \times 100 /$ Drug loading (theoretical) $(\%)$.

Abbreviations: NA, not applicable; PLGA, poly(d,I-lactic-co-glycolic acid).

was stirred at $130 \mathrm{rpm}$ in a rotary vacuum evaporator (PBU-6; Superfit, Mumbai, India) with a temperature-controlled water bath $\left(40^{\circ} \mathrm{C}\right)$ for 30 minutes for quick removal of the organic solvent (DCM). The emulsion was kept on a magnetic stirrer overnight for complete removal of the DCM. The next day, any particles that had formed were collected by centrifugation at 15,000 rpm for 1 hour. To remove free drug, polymeric particles were resuspended in water and centrifuged three times at 15,000 rpm for 30 minutes each. Particles were then freeze-dried (by prefreezing at $-20^{\circ} \mathrm{C}$ overnight and lyophilizing at $-40^{\circ} \mathrm{C}$ for 12 hours) in a lyophilizer (laboratory lyophilizer; IIC Industrial Corporation, Kolkata, India) and stored at $4^{\circ} \mathrm{C}$. Formulations S1, S2, and S5 were prepared as described above, except that $\mathrm{S} 3$ and $\mathrm{S} 4$ were centrifuged at $16,000 \mathrm{rpm}$ and no drug was added to S5.

\section{Preparation of gold nanoparticles}

Gold nanoparticles were prepared by citrate reduction of $\mathrm{HAuCl}_{4}$ following the methods of Storhoff et al. ${ }^{7}$ All glasswares were first cleaned in aqua regia (three parts $\mathrm{HCl}$, one part $\mathrm{HNO}_{3}$ ), rinsed with nanopure water, and dried in a hot air oven (Orion Industries, Kalka, India). An aqueous solution of $\mathrm{HAuCl}_{4}(1 \mathrm{mM}, 500 \mu \mathrm{L})$ was brought to boiling while stirring continuously with a glass rod, and the entire $50 \mathrm{~mL}$ aliquot of $38.8 \mathrm{mM}$ trisodium citrate solution was quickly added, which resulted in a color change of the solution from pale yellow to deep red. After the color change, the solution was allowed to cool and was subjected to high-speed centrifugation (3K30 Sigma Lab Centrifuge; Merrington Hall Farm, Shrewsbury, UK) at $12,800 \mathrm{rpm}$ at $4^{\circ} \mathrm{C}$ for 20 minutes. The gold nanoparticle pellet was then resuspended in water at $\mathrm{pH} 7.0$ after discarding the supernatant. The process was repeated three times to eliminate the free citrate. ${ }^{8}$

\section{Preparation of gold and drug-loaded polymeric nanoparticles}

PVA solutions were prepared in 1.5\% (by dissolving $1.5 \mathrm{~g}$ PVA in $100 \mathrm{~mL}$ water) and $2.5 \%$ (by dissolving $0.25 \mathrm{~g}$ of PVA in $10 \mathrm{~mL}$ water) concentrations. Colloidal gold solution $(1 \mathrm{~mL})$ was added to the $2.5 \%$ PVA solution. PLGA (250 mg) was dissolved in $2 \mathrm{~mL}$ DCM. The total amount of stavudine (Table 1) was dissolved in $0.5 \mathrm{~mL}$ of the $2.5 \%$ PVA-gold mixture. This drug solution $(0.5 \mathrm{~mL})$ was added drop-wise into $2 \mathrm{~mL}$ of the PLGA solution, and the mixture was homogenized at different speeds for different formulations (Table 1) for 4 minutes. The preparation method was then continued as described previously. To remove uncoated gold nanoparticles from suspension after encapsulation, the mixture was centrifuged at $6000 \mathrm{rpm}$ for 1 hour. The supernatant was decanted carefully, and coated nanoparticles were separated from the supernatant by centrifugation at 15,000 rpm for 1 hour and then washed three times by centrifuging at 15,000 rpm and resuspending in water. Formulations S2 and $\mathrm{S} 4$ were prepared as described above, except that S4 was centrifuged at $16,000 \mathrm{rpm}$.

\section{Nanoparticles with FITC}

Stavudine-loaded nanoparticles were prepared with a fluorescent probe (FITC) to study the uptake of nanoparticles by specific cells. FITC-loaded nanoparticles were prepared essentially as described above, except that an FITC stock solution $(0.4 \%$ weight $[\mathrm{w}] /$ volume [v] FITC in absolute ethanol) of $100 \mu \mathrm{L}$ was added to the polymeric phase during the preparation of the primary emulsion. The ratio of the fluorophore to nanoparticles taken was 1:80. Preparation was then continued as described above.

\section{Evaluation and characterization of stavudine-loaded nanoparticles Drug-excipient interactions by Fourier transform infrared spectroscopy (FTIR)}

Stavudine, PLGA, PVA, gold nanoparticles, PLGA-PVA combination, stavudine-PLGA-PVA combination, and stavudine-PLGA-PVA-gold nanoparticles combination were mixed with infrared-grade potassium bromide $(\mathrm{KBr})$ 
and compressed into pellets by applying $5.5 \mathrm{t}$ of pressure in a hydraulic press. The pellets were scanned over a wavenumber range of $4000 \mathrm{~cm}^{-1}$ to $400 \mathrm{~cm}^{-1}$ in an FTIR spectrometer (MAGNA-IR 750; Nicolet Instruments Corporation, Madison, WI).

\section{Morphology of nanoparticles by field-emission scanning electron microscopy (FESEM)}

The external morphology of nanoparticles of different formulations was analyzed by FESEM. The freeze-dried particles were spread onto metal stubs and platinum coating applied by using an ion-sputtering device. The coated particles were then examined under FESEM (JSM 6100; JEOL, Tokyo, Japan).

\section{Determination of presence of gold in polymeric nanoparticle by energy dispersive $\mathrm{X}$-ray spectroscopy (EDX)}

EDX was part of the FESEM system. SEM-EDX, which uses an SEM system, helps determine the chemical composition of a specimen, and we used this to determine the presence of gold in stavudine-gold-loaded polymeric nanoparticles.

\section{Determination of size distribution, polydispersity index (PDI), and zeta potential}

Size distribution, PDI, and zeta potential were measured by a Zetasizer Nano ZS (0.6 nm to $6000 \mathrm{~nm}$ ) with DTS software version 4.0 (Malvern Instruments Ltd, Malvern, UK). The freeze-dried formulations were suspended in double-distilled water and then poured into a glass cuvette and analyzed. For particle size measurement, dynamic light scattering is used, and the software collects and interprets data on particle size and zeta potential and calculates the average size and PDI by using the intensity, volume, and number distribution. Mean particle diameter was calculated from the measured size distributions, and the PDI was calculated based on the size range present in the suspension as determined in a Zetasizer Nano ZS (Malvern Instruments Ltd).

\section{Drug content and entrapment efficiency study}

Exactly $2 \mathrm{mg}$ of each product sample was placed in separate $2 \mathrm{~mL}$ Eppendorf microcentrifuge tubes. A prepared solution of $5 \% \mathrm{w} / \mathrm{v}$ sodium dodecyl sulfate in $1 \mathrm{~mL} 0.1 \mathrm{M} \mathrm{NaOH}$ solution was added to each tube with a micropipette. The tubes were placed in an incubator shaker (Somax Incubator Shaker; Shenzhen Pango Electronic Co, Ltd, Shenzhen, China) at 120 rpm for 3 hours at $37^{\circ} \mathrm{C}$. The tubes were then centrifuged for
10 minutes at $5000 \mathrm{rpm}$ and the supernatant liquid collected with a micropipette. The absorbance of drug in solution was read with an ultraviolet absorption spectroscope (Beckman Instruments, Fullerton, CA) at $266 \mathrm{~nm}$ against a blank containing $5 \% \mathrm{w} / \mathrm{v}$ sodium dodecyl sulfate in $0.1 \mathrm{M} \mathrm{NaOH}$. Drug concentrations were determined from the calibration curve. The drug-loading and drug entrapment efficiencies were calculated using the following formulae:

Drug loading (theoretical) $(\%)$

$=\frac{\text { Amount of drug taken to prepare nanoparticles } \times 100}{\text { Amount of PLGA }+ \text { drug taken }}$

Drug loading (actual) $(\%)$

$$
\begin{gathered}
=\frac{\text { Amount of drug in nanoparticles } \times 100}{\text { Amount of nanoparticles obtained }} \\
\text { Drug entrapment efficiency }(\%) \\
=\frac{\text { Drug loading (actual) }(\%) \times 100}{\text { Drug loading (theoretical) }(\%)} .
\end{gathered}
$$

\section{Drug release study}

To determine drug release at the different time points, $5 \mathrm{mg}$ stavudine-loaded nanoparticles of different formulations were suspended in $1 \mathrm{~mL}$ phosphate-buffered saline ([PBS] $\mathrm{pH}$ 7.4) in prelabeled microcentrifuge tubes and kept in an incubator shaker (Somax Incubator Shaker) at $37^{\circ} \mathrm{C}$ with constant shaking at $72 \mathrm{rpm}$ after brief vortexing. Each formulation was processed in triplicate, and samples were kept for specific periods of time up to 63 days. At any particular time point, only the sample for analysis was removed from the shaker, centrifuged at $15,000 \mathrm{rpm}$ for $30 \mathrm{~min}$ at $4^{\circ} \mathrm{C}$, and drug from the supernatant was analyzed at a wavelength of $266 \mathrm{~nm}$ with a UV-Vis spectrophotometer (Beckman Instruments). The percentage of drug release was calculated as:

Drug release $(\%)$

$$
=\frac{\text { Amount of drug released }}{\text { Amount of drug loaded in } 5 \mathrm{mg} \text { of nanoparticle }} \times 100
$$

\section{Drug release kinetics study}

To understand the release kinetics, data obtained from in vitro drug release studies were plotted in various kinetic models: zero order as cumulative amount of drug released versus time; first order as logarithmic value of cumulative percentage of drug remained versus time; Higuchi model as cumulative percentage of drug released versus square root 
of time; Korsmeyer-Peppas model as logarithmic value of cumulative percentage of drug released versus logarithmic value of time; and Hixson-Crowell model as cube root percentage drug remained versus time. ${ }^{16}$ The in vitro drug release data were also fitted to the Hopfenberg model. ${ }^{17,18}$

\section{In vitro cellular uptake study}

Macrophages at a density of $2.5 \times 10^{5}$ cells $/ \mathrm{mL}$ were suspended in serum-free Roswell Park Memorial Institute 1640 medium (Sigma-Aldrich Corporation). ${ }^{15}$ A cell suspension of $200 \mu \mathrm{L}$ was placed in eight-well tissue culture plates, each well of which contained $800 \mu \mathrm{L}$ medium. Cells were allowed to adhere for 2 hours in a $\mathrm{CO}_{2}$ incubator with a supply of $5 \% \mathrm{CO}_{2}$ at $37^{\circ} \mathrm{C}$. The medium was then removed, and the wells were washed twice with serum-free medium. The adherent macrophages were incubated with FITC-stavudinenanoparticles (S4) at different concentrations $(300 \mathrm{ng} / \mathrm{mL}$ and $500 \mathrm{ng} / \mathrm{mL}$ of medium) in $500 \mu \mathrm{L}$ of fresh medium. After 4 hours, wells containing macrophages were washed twice with serum-free medium, observed under confocal laser scanning microscopy (LSM MAT 510; Carl Zeiss, Jena, Germany), and photographs taken.

Intensity of the fluorescence of the individual macrophages was quantified with a defined line and color region, analyzed using Zen software (Zen Software Ltd, Manchester, UK), and fluorescence intensity histograms generated (not shown). Image software was used to correct threshold and background levels ${ }^{19}$ and to quantify the degree of fluorescence ${ }^{20}$ and the quantity of nanoparticles in cells.

The quantity of gold nanoparticles within the cells was determined using the atomic emission spectroscopy of aciddigested cells. ${ }^{21}$ The method ensures a highly sensitive analysis, and a comparison of gold nanoparticle uptake by all exposed cells has been successfully used to quantify the uptake of the unmodified gold nanoparticle in the range of parts per billion. At 4 hours incubation time, cell aliquots were washed twice with cold PBS ( $\mathrm{pH} 7.4$ ) by centrifugation at $4000 \mathrm{rpm}$ for 10 minutes at $4^{\circ} \mathrm{C}$ and resuspended in cold PBS. The pellet was then collected and analyzed using the method described. ${ }^{21}$

\section{Results}

Drug-excipient interactions provide information on the stability of the drug in formulation, the drug-release pattern from the formulation, and the lag time of drug release. ${ }^{10}$ Among the various methods available, FTIR spectroscopy provides a methodology for examining interactions between various functional groups present in drug and excipients. The FTIR spectrum of the pure drug and individual FTIR spectrum of
PLGA, PVA, PVA-PLGA, and PVA-PLGA-drug are shown in Figure 1. Comparing the results in Figure 1A with those of Figure 1B-E, it can be seen that there was minor shifting of some of the peaks between wavenumbers $3600 \mathrm{~cm}^{-1}$ and $2800 \mathrm{~cm}^{-1}, 1700 \mathrm{~cm}^{-1}$ and $1800 \mathrm{~cm}^{-1}$, and $750 \mathrm{~cm}^{-1}$ and $500 \mathrm{~cm}^{-1}$. Wavenumber $3600 \mathrm{~cm}^{-1}$ to $3800 \mathrm{~cm}^{-1}$ is the infrared absorption frequency stretching vibration zone of strong intensity $\mathrm{CH}_{3}, \mathrm{CH}_{2}, \mathrm{CH}$, medium intensity $=\mathrm{CH}$ and $=\mathrm{CH}_{2}$, strong intensity $\mathrm{OH}$ (hydrogen bonded), variable intensity $\mathrm{OH}$ (free), medium intensity $\mathrm{CH}$ (aldehyde), and weak intensity $\mathrm{NH}\left(1^{\circ}\right.$ amine $)$ and $\mathrm{NH}\left(2^{\circ}\right.$ amine $){ }^{22}$ Wave number $1700 \mathrm{~cm}^{-1}$ to $1800 \mathrm{~cm}^{-1}$ is the strong intensity stretching vibration zone of $\mathrm{C}=\mathrm{O}$ (saturated aldehyde), $\mathrm{C}=\mathrm{O}$ (saturated ketone), cyclopentanone, and cyclobutanone. ${ }^{22}$ Wave number $750 \mathrm{~cm}^{-1}$ to $500 \mathrm{~cm}^{-1}$ is the bending vibration zone of weak intensity $\mathrm{CH}_{2}$ rocking, medium intensity out of plane $=\mathrm{CH}_{2}$, medium intensity cis $-\mathrm{RCH}=\mathrm{CHR}$, strong intensity $\mathrm{CH}$ deformation, strong to medium intensity $\mathrm{CH}$ bending and ring puckering, weak $\mathrm{OH}$ out of plane bending, and variable $\mathrm{NH}_{2}$ and $\mathrm{NH}$ wagging.

In stavudine, $\mathrm{CH}_{3}, \mathrm{C}=\mathrm{O}, \mathrm{NH}, \mathrm{N}, \mathrm{OH}$ are the reactive groups, whereas in $\mathrm{PLGA}, \mathrm{CH}_{3}, \mathrm{C}=\mathrm{O}$, and $=\mathrm{O}$ reactive groups are present. For PVA, $\mathrm{OH}$ and $\mathrm{H}$ are the predominant reactive groups. Thus, there might be some physical interactions between the functional groups of the drug and the excipients that resulted in a minor shifting of the peaks. Formation of a weak hydrogen bond, interaction due to van der Waals force, or dipole-dipole interaction among these functional groups might be responsible for these physical interactions. The presence of the characteristic peaks of the drug and the excipients suggests that there was no chemical interaction. Further, FTIR spectra of the drug with gold mixture and of the drug with gold and other excipients showed no shifting of the characteristic peaks (data not shown). Thus, FTIR spectroscopic data suggest that there was no chemical interaction between the drug and the excipients, indicating that the polymer is suitable for formulation of the anti-AIDS drug. However, a few physical interactions occurred among some functional groups of the drug and the excipients. These might have been responsible for sustained release of the drug from the formulations and might provide information on the spherical structure of the nanoparticles.

Particles were mostly in the nanoscale range with a smooth surface; however, there were some particles larger than nanosize (Figure 2). The Z-average of S1-S5 formulations were $1365 \mathrm{~nm}, 1136 \mathrm{~nm}, 211 \mathrm{~nm}, 201 \mathrm{~nm}$, and $2038 \mathrm{~nm}$, respectively (Table 2); polydispersity indices of the S1-S5 formulations were $0.102,0.109,0.424,0.376$, and 0.046, respectively. Particle size distribution (by percentage intensity) of the 
A

Stavudine

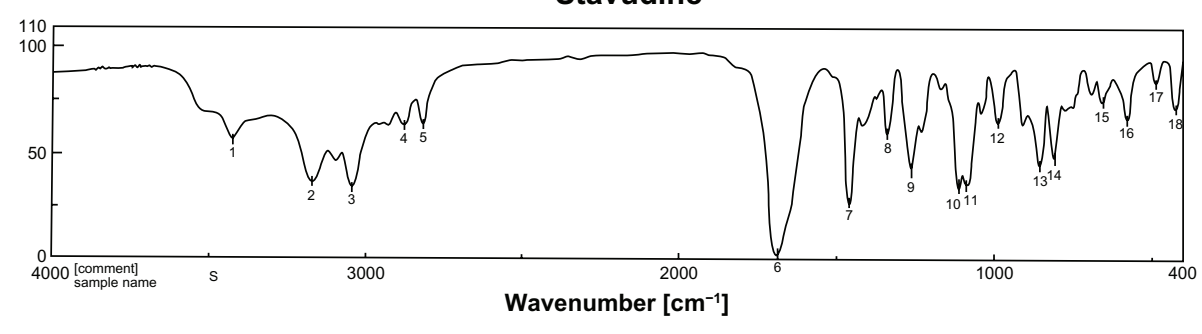

B

PLGA

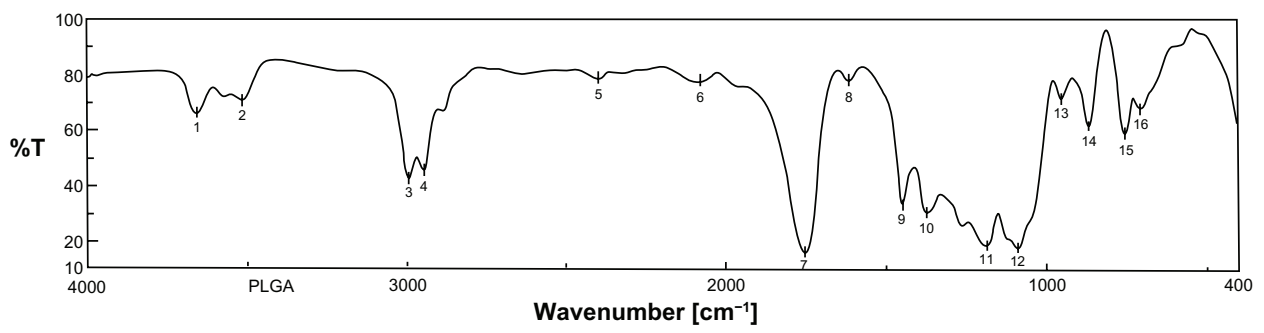

C

PVA

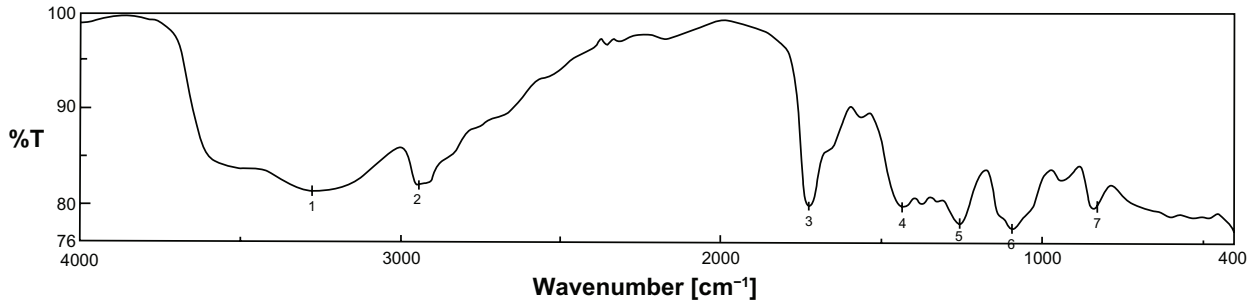

D Mixture of PLGA and PVA

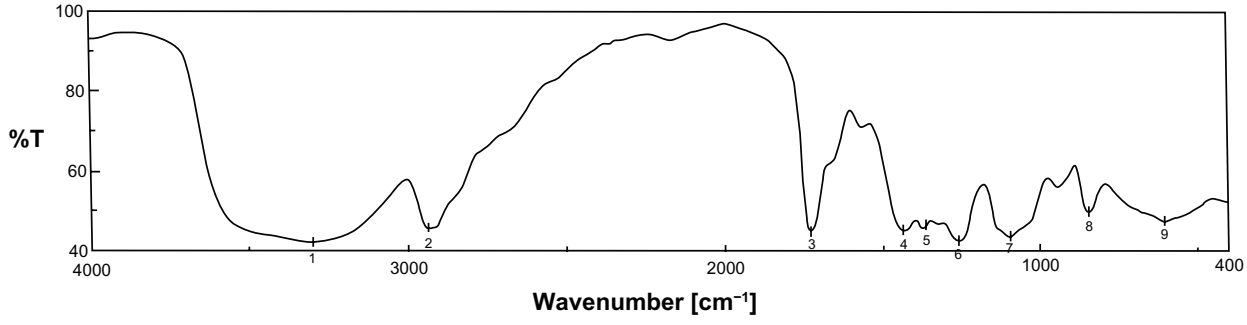

E

Stavudine, PLGA and PVA

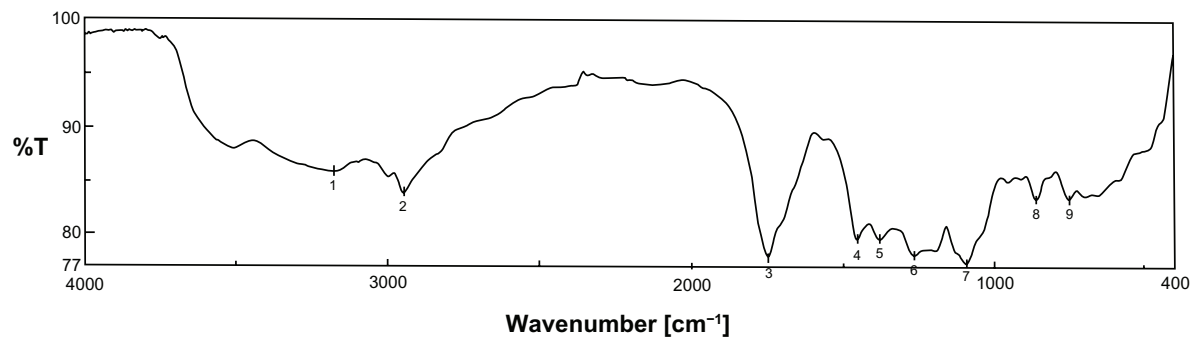

Figure I FTIR spectra of (A) stavudine; (B) PLGA; (C) PVA; (D) PVA and PLGA mixture; and (E) stavudine, PVA, and PLGA mixture.

Abbreviations: FTIR, Fourier transform infrared spectroscopy; PLGA, poly(d,I-lactic-co-glycolic acid); PVA, polyvinyl alcohol.

experimental formulations are shown in Figure 3. Zeta potentials of S1-S5 were $-12.6,-8.16,-27.8,-19.6$, and $-17 \mathrm{mV}$, respectively (Table 2). The dispersant refractive index, viscosity, and dispersant (water) dielectric constant were 1.33, 0.8872 , and 78.5 , respectively, at $25^{\circ} \mathrm{C}$. The incorporation of gold in nanoparticles was assessed from the EDX data, which showed the presence of gold particles in gold-encapsulated polymeric nanoparticles (Figure 4) as compared to gold nanoparticles (Figure 5) alone.

Maximum conductivity was recorded for S2 (0.034), followed by S1 (0.011) and S5 (0.012) (Table 2). However, conductivities were only slightly lower for S3 and S4. 


\section{A}

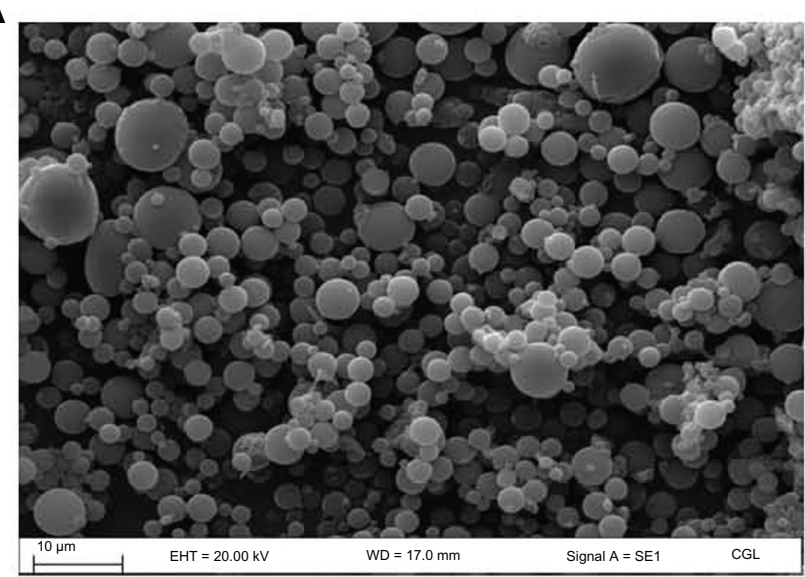

B
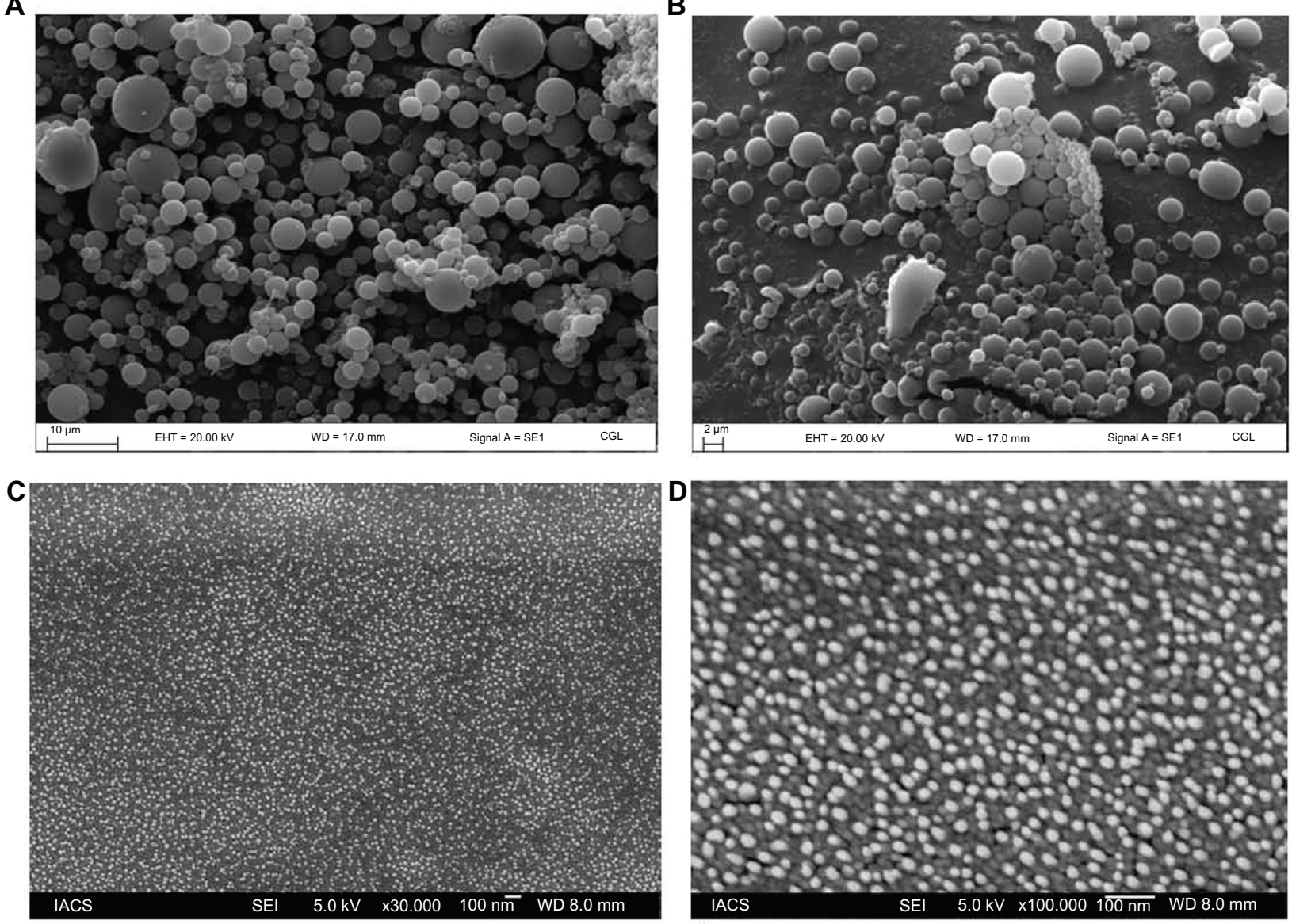

\section{$\mathrm{E}$}
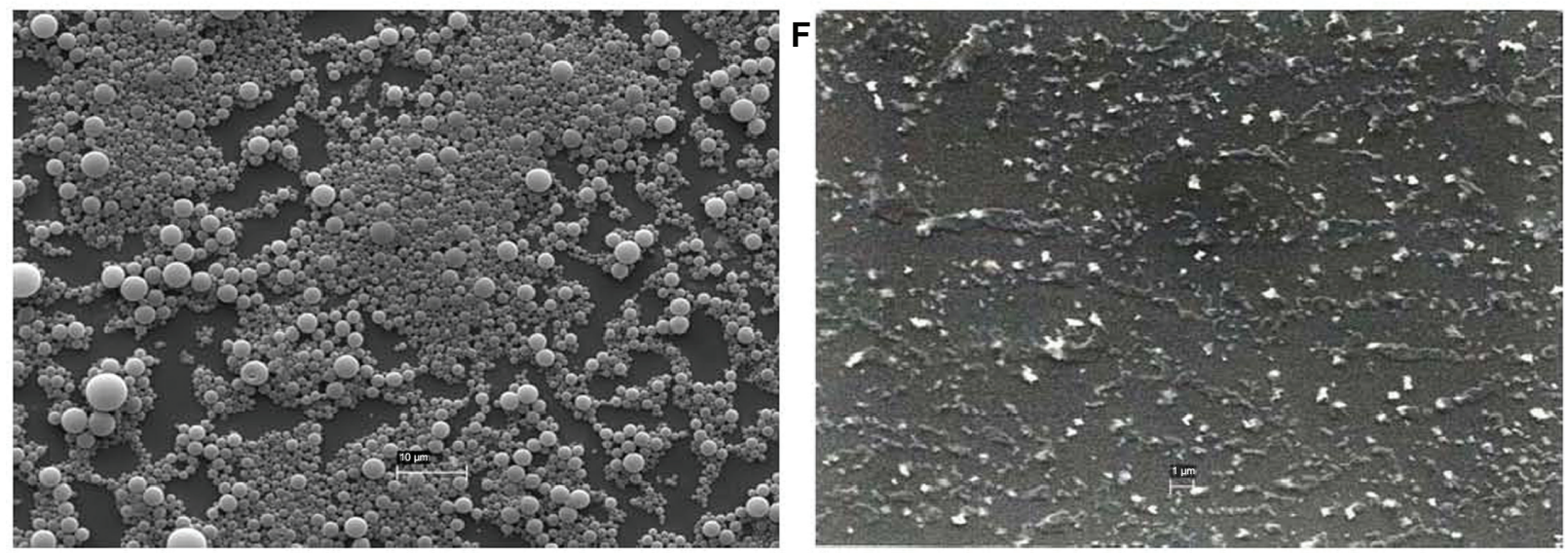

Figure 2 FESEM photographs of formulations. (A) Formulation SI prepared at 15,000 rpm shows that the particles were of micrometer sizes. (B) Formulation S2 prepared at 15,000 rpm shows that the particles were in micron range. (C) Formulation S3 prepared at 16,000 rpm shows that the particles were in nanometer range. (D) Formulation S4 prepared at 16,000 rpm shows that the particles were in nanometer range. (E) Formulation S5 prepared at 15,000 rpm shows that the particles were mostly in micrometer range. (F) SEM photograph of the gold nanoparticles shows that the particles were in submicron range with variable sizes.

Abbreviations: FESEM, field-emission scanning electron microscopy; SEM, scanning electron microscopy.

The drug-loading values were expressed in terms of the quantity of drug entrapped in the formulations, ${ }^{11}$ and the drug entrapment efficiency was associated with the percentage of drug entrapped with respect to theoretical drug loading in a particular formulation. S3 had the highest drug content and drug entrapment efficiency among the formulations S1 to S4 (Table 1).
The in vitro drug release profile of stavudine from the PLGA-based particulate drug formulations is shown in Figure 6 . The cumulative amount of drug release varied between $65 \%$ and $72 \%$ of the actual drug content in the formulations for 63 days. Gold along with stavudine-loaded polymeric nanoparticles (S4) prepared at a higher homogenization 
Table 2 Size distribution (Z-average [nm]), polydispersity index, zeta potential, and conductivity of experimental nanoparticulate formulations

\begin{tabular}{lllll}
\hline $\begin{array}{l}\text { Formulation } \\
\text { code }\end{array}$ & $\begin{array}{l}\text { Z-average } \\
(\mathbf{n m})\end{array}$ & PDI & $\begin{array}{l}\text { Zeta potential } \\
(\mathbf{m V})\end{array}$ & $\begin{array}{l}\text { Conductivity } \\
(\mathbf{m S} / \mathbf{c m})\end{array}$ \\
\hline SI & 1365 & 0.102 & -12.6 & 0.0117 \\
S2 & 1136 & 0.109 & -8.16 & 0.0346 \\
S3 & 211 & 0.424 & -27.8 & 0.00894 \\
S4 & 201 & 0.376 & -19.6 & 0.00882 \\
S5 & 2038 & 0.046 & -17 & 0.0125 \\
\hline
\end{tabular}

Abbreviation: PDI, polydispersity index.

speed $(16,000 \mathrm{rpm})$ showed a higher percentage of drug release in 63 days compared to $\mathrm{S} 3$ during the same time period.

Data related to drug release as assessed by different kinetic models ${ }^{16}$ showed more linearity in $\mathrm{R}^{2}$ values (Table 3 ) in Higuchi, Korsmeyer-Peppas, and Hopfenberg plots. This suggests anomalous diffusion of drug as well as erosion of the matrix type formulations.

Based on the size and drug-loading in gold containing PLGA nanoparticles, S4 was selected for the nanoparticle uptake study. Nanoparticle uptake by macrophages was observed in vitro after treatment of nanoparticles at two different concentrations for 4 hours (Table 4; Figure 7). As the nanoparticle concentration increased, nanoparticle uptake increased. About $34 \%$ and $45 \%$ of the nanoparticles were internalized in 4 hours at nanoparticle doses of $300 \mathrm{ng} / \mathrm{mL}$ (gold content $450 \mathrm{pg} / 0.5 \times 10^{5}$ cells) and $500 \mathrm{ng} / \mathrm{mL}$ (gold content $867 \mathrm{pg} / 0.5 \times 10^{5}$ cells), respectively, as assessed by fluorescence intensity.

\section{Discussion}

Stavudine-loaded PLGA nanoparticles and stavudine with colloidal gold loaded in PLGA nanoparticles were presented in this research work. PLGA nanoparticles were formulated using an emulsification solvent evaporation technique. PVA was selected as an effective stabilizer ${ }^{12}$ and was used to stabilize the emulsion since it also helps form relatively small and uniform-sized particles. ${ }^{13,14}$

FTIR spectroscopy was used for the drug-excipients interaction study to identify interactions ${ }^{10}$ at the functional group level. No chemical interaction was detected between the drug and the excipients since there was no shifting of individual characteristic peaks. However, a few physical interactions were found among some functional groups of the drug and the excipients, and these interactions might be responsible for the spherical structure of the nanoparticles and their sustained drug release.
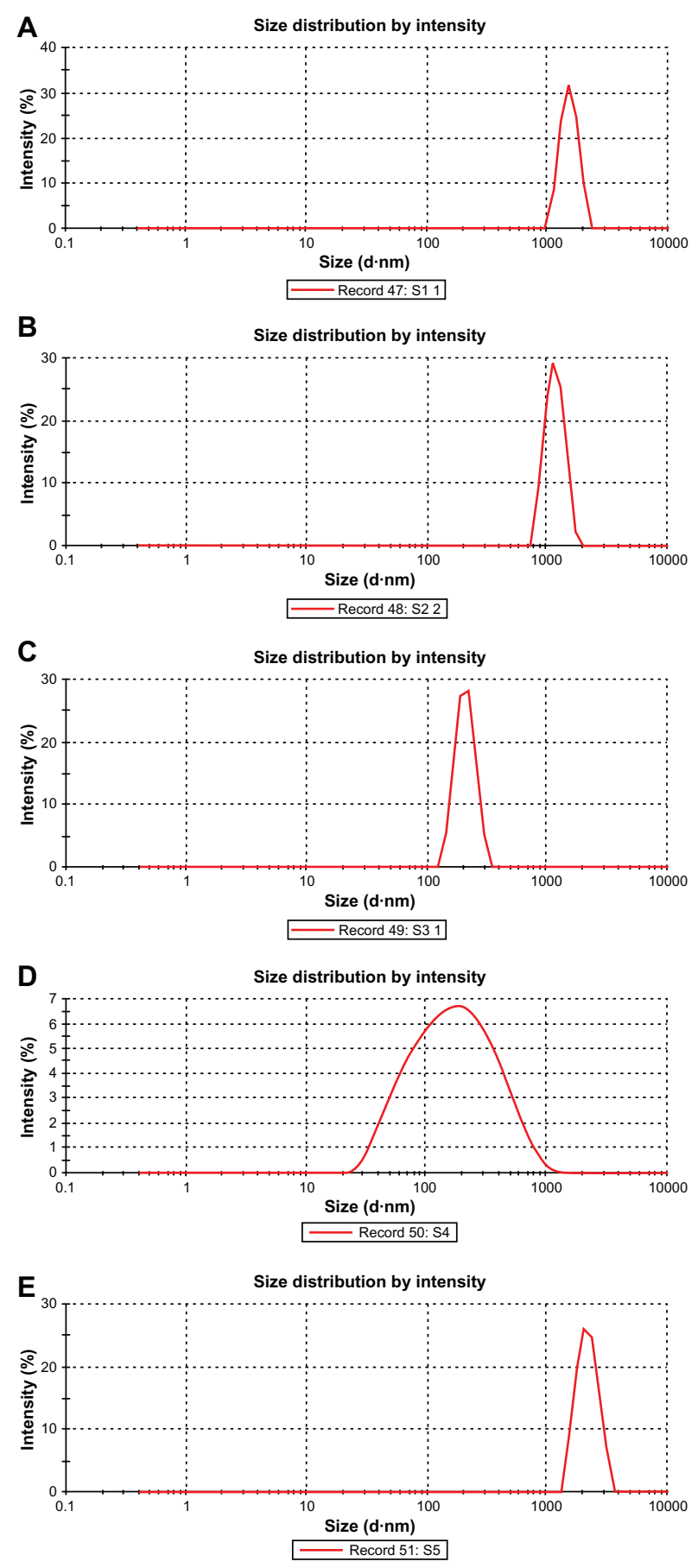

Figure 3 Size distribution of different formulations. (A) SI (by intensity); (B) S2 (by intensity); (C) S3 (by intensity); (D) S4 (by intensity); (E) S5 (by intensity).

The experimental particles have smooth surface, and particles prepared at 15,000 rpm were larger compared to particles prepared at $16,000 \mathrm{rpm}$. The data suggest that increasing homogenization speed results in decreases to average particle diameters. Particles prepared at higher homogenizing speed were in the nanoscale range and were 


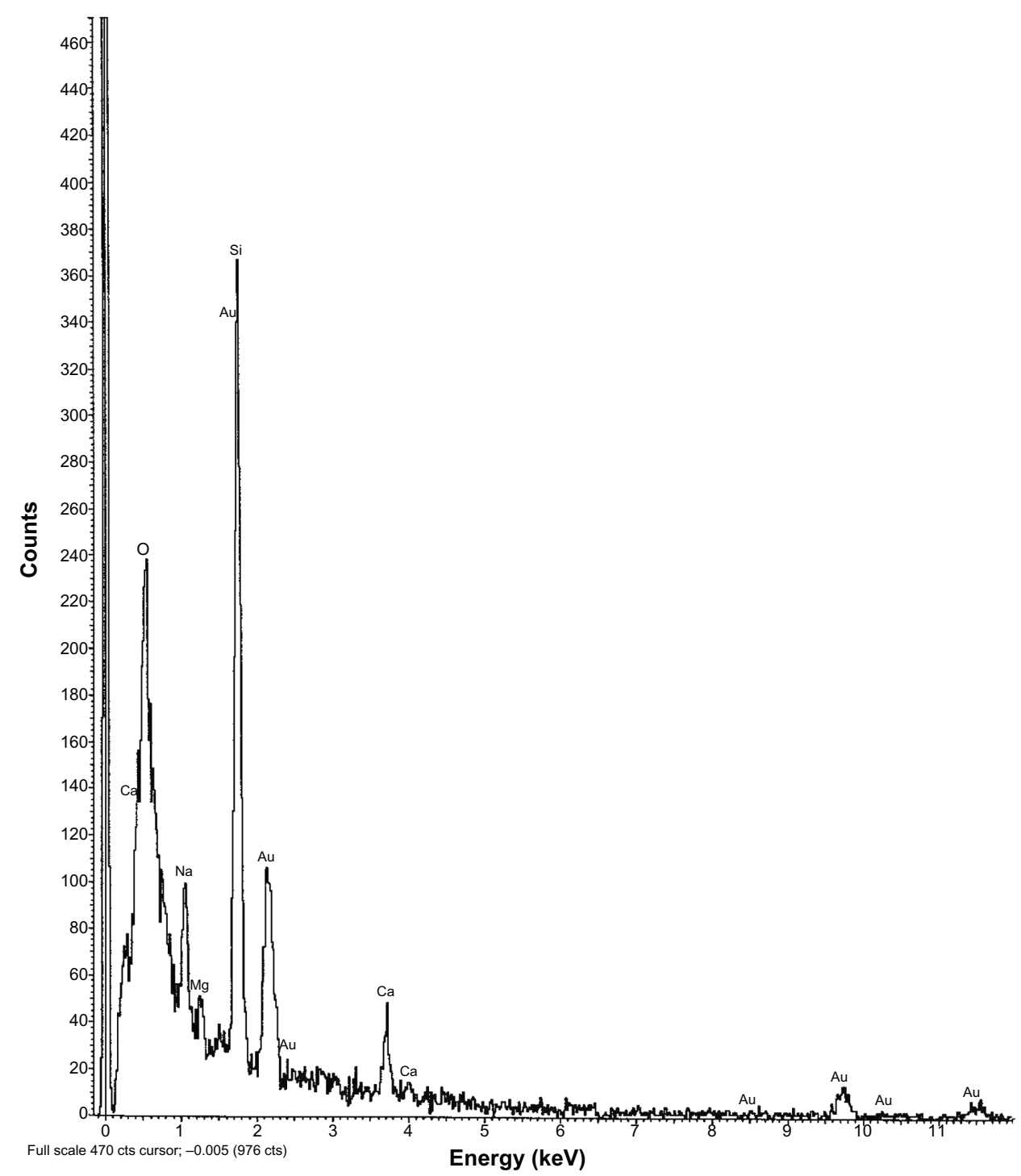

Figure 4 EDX data showing the presence of gold particles in gold-encapsulated polymeric nanoparticles.

Abbreviation: EDX, energy dispersive $\mathrm{X}$-ray spectroscopy.

homogeneously distributed. Encapsulation of colloidal gold reduced the particle size even further. The incorporation of gold in the nanoparticles was confirmed by the EDX data.

An earlier study showed that polydispersity variation is present from $100 \mathrm{~nm}$ to as large as $20 \mu \mathrm{m} .{ }^{23}$ In our study, polydispersity indices of the formulations varied widely. The maximum PDI value was obtained for S5. The PDI value was greater for all formulations with gold particles, suggesting that the value was enhanced due to the presence of the cationic metal.

Particles had negative zeta potentials. The resulting negative charges were caused by the dissociation of the hydrogen ion from the carboxyl group $(-\mathrm{COOH})$ in the PLGA chain.
Addition of PVA to the formulation also conveyed a negative zeta potential to the resulting nanoparticle, further influencing both particle stability and the cellular uptake ability of the nanoparticles. ${ }^{24,25}$ The presence of gold particles lowered the zeta potentials of the formulation since gold is cationic. ${ }^{26}$ Particles with zeta potentials greater than $+30 \mathrm{mV}$ and less than $-30 \mathrm{mV}$ are normally considered to form stable colloidal dispersion. ${ }^{10}$ If the magnitude of the zeta potential decreases, particle aggregation may occur, and this would cause a rapid precipitation of suspended nanoparticles. Our results indicate that all the formulations were unstable in terms of rapid precipitation in the colloidal state. The zeta potential values suggest that $\mathrm{S} 3$ had greater stability compared to the others in the dispersion state. S2 was the least stable in terms of 


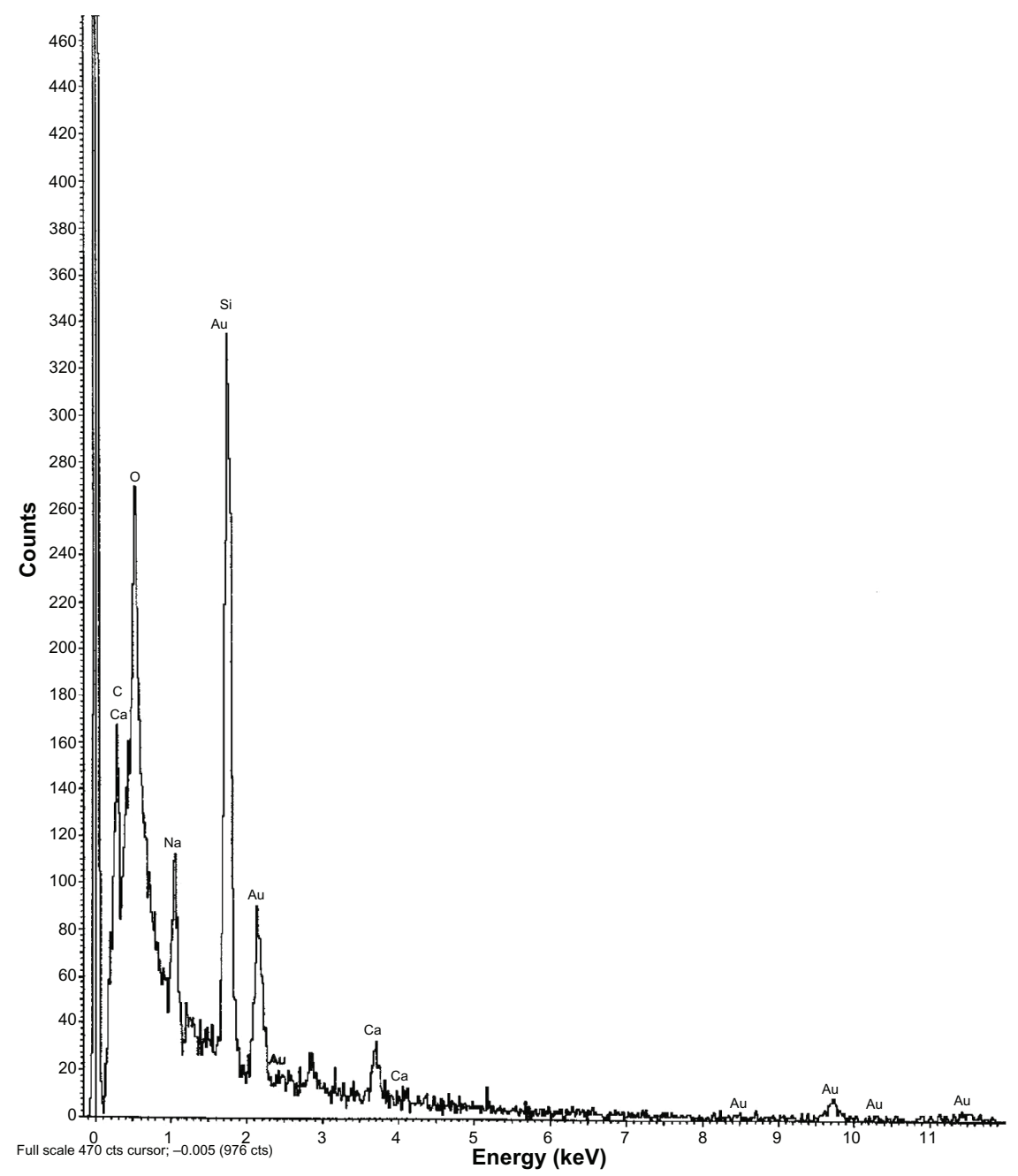

Figure 5 EDX data of colloidal gold nanoparticles alone.

Abbreviation: EDX, energy dispersive X-ray spectroscopy.

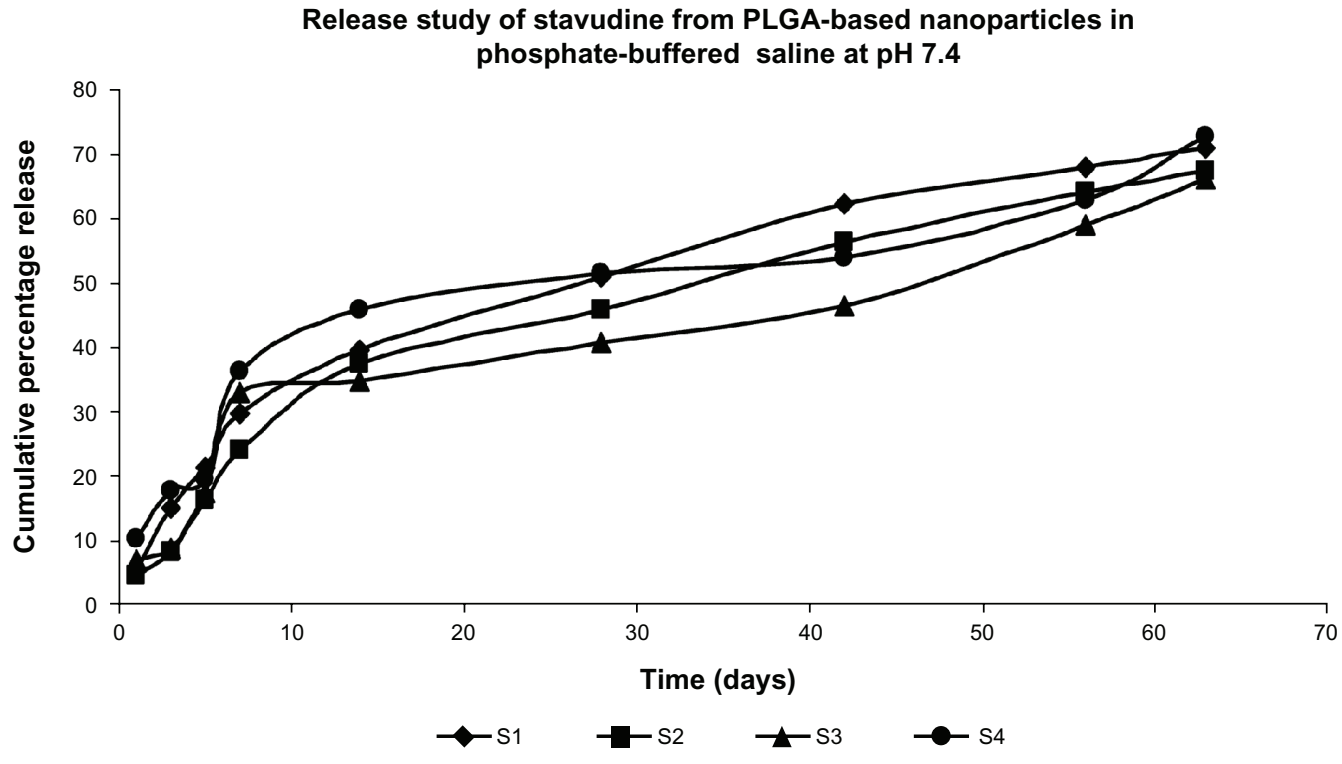

Figure 6 Release profile of didanosine from different formulations in phosphate-buffered saline ( $\mathrm{pH}$ 7.4). Note: Data show means $(n=3)$. 
Table 3 Data of drug release kinetics of various formulations

\begin{tabular}{|c|c|c|c|c|}
\hline Kinetic model & Formulation SI & Formulation S2 & Formulation S3 & Formulation S4 \\
\hline \multirow[t]{2}{*}{ Zero order } & $R^{2}=0.890$ & $R^{2}=0.917$ & $R^{2}=0.886$ & $\mathrm{R}^{2}=0.855$ \\
\hline & $\mathrm{K}_{0}\left(\mu \mathrm{g} \mathrm{mL}^{-1} \mathrm{~h}^{-1}\right)=1.0257$ & $\mathrm{~K}_{0}\left(\mu \mathrm{gL}^{-1} \mathrm{~h}^{-1}\right)=0.9542$ & 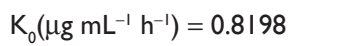 & $\mathrm{K}_{0}\left(\mu \mathrm{g} \mathrm{mL}^{-1} \mathrm{~h}^{-1}\right)=0.834 \mathrm{I}$ \\
\hline \multirow{2}{*}{ First order } & $R^{2}=0.966$ & $\mathrm{R}^{2}=0.973$ & $\mathrm{R}^{2}=0.93 \mathrm{I}$ & $\mathrm{R}^{2}=0.908$ \\
\hline & $\mathrm{K}_{1}\left(\mathrm{~h}^{-1}\right)=-0.008 \mathrm{I}$ & $\mathrm{K}_{1}\left(\mathrm{~h}^{-1}\right)=-0.0072$ & $\mathrm{~K}_{1}\left(\mathrm{~h}^{-1}\right)=-0.0063$ & $K_{1}\left(h^{-1}\right)=-0.0072$ \\
\hline \multirow{2}{*}{ Higuchi } & $\mathrm{R}^{2}=0.986$ & $\mathrm{R}^{2}=0.984$ & $\mathrm{R}^{2}=0.95 \mathrm{I}$ & $\mathrm{R}^{2}=0.945$ \\
\hline & $\mathrm{K}_{\mathrm{H}}\left(\mathrm{h}^{-1 / 2}\right)=9.2194$ & $\mathrm{~K}_{\mathrm{H}}\left(\mathrm{h}^{-1 / 2}\right)=8.9496$ & $\mathrm{~K}_{\mathrm{H}}\left(\mathrm{h}^{-1 / 2}\right)=7.859 \mathrm{I}$ & $\mathrm{K}_{\mathrm{H}}\left(\mathrm{h}^{-1 / 2}\right)=8.4073$ \\
\hline \multirow{2}{*}{ Korsmeyer-Peppas } & $\mathrm{R}^{2}=0.825$ & $\mathrm{R}^{2}=0.963$ & $\mathrm{R}^{2}=0.919$ & $\mathrm{R}^{2}=0.901$ \\
\hline & $\mathrm{n}=0.785$ & $n=0.657$ & $\mathrm{n}=0.519$ & $\mathrm{n}=0.453$ \\
\hline \multirow[t]{2}{*}{ Hixson-Crowell } & $R^{2}=0.945$ & $R^{2}=0.958$ & $R^{2}=0.918$ & $R^{2}=0.889$ \\
\hline & $\mathrm{K}_{\mathrm{HC}}\left(\mu \mathrm{g}^{1 / 3} \mathrm{t}^{-1}\right)=-0.0233$ & $\mathrm{~K}_{\mathrm{HC}}\left(\mu \mathrm{g}^{1 / 3} \mathrm{t}^{-1}\right)=-0.0212$ & $\mathrm{~K}_{\mathrm{HC}}\left(\mu \mathrm{g}^{1 / 3} \mathrm{t}^{-1}\right)=-0.0189$ & $\mathrm{~K}_{\mathrm{HC}}\left(\mu \mathrm{g}^{1 / 3} \mathrm{t}^{-1}\right)=-0.02 \mathrm{I}$ \\
\hline \multirow[t]{2}{*}{ Hopfenberg } & $R^{2}=0.985 I$ & $R^{2}=0.9679$ & $R^{2}=0.9143$ & $\mathrm{R}^{2}=0.9199$ \\
\hline & $\mathrm{K}\left(\mu \mathrm{g} \mathrm{mg} \mathrm{g}^{-1} \mu \mathrm{m}^{-1}\right) \mathrm{h}^{-1}=0.0092$ & $\mathrm{~K}\left(\mu \mathrm{g} \mathrm{mg}^{-1} \mu \mathrm{m}^{-1}\right) \mathrm{h}^{-1}=0.0104$ & $\mathrm{~K}\left(\mu \mathrm{g} \mathrm{mg^{-1 }} \mu \mathrm{m}^{-1}\right) \mathrm{h}^{-1}=0.0559$ & $\mathrm{~K}\left(\mu \mathrm{g} \mathrm{mg^{-1 }} \mu \mathrm{m}^{-1}\right) \mathrm{h}^{-1}=0.1139$ \\
\hline
\end{tabular}

forming a stable suspension, and these formulations should be stored in a lyophilized condition and reconstituted in an aqueous vehicle immediately before use.

Gold nanoparticles have a strong affinity toward sulfur of the sulfhydryl (-SH) group through covalent interaction. However, the most intriguing factor is the interaction between amine and gold nanoparticles ${ }^{19}$ as this type of interaction makes the gold nanoparticles more flexible upon attachment and release. Any electronic perturbation can tune the attachment or release of the amine-containing molecule (ie, the drug) from the gold nanoparticle surface. ${ }^{26}$ In the present study drug molecules were released relatively faster from the PLGA nanoparticles containing gold. There was no physicochemical interaction detected between the drug and the gold nanoparticles, and the presence of gold in the PLGA nanoparticles reduced the size (Z-average) of the particles. Thus, the presence of gold in PLGA nanoparticles might have shortened the length of the drug diffusion pathways thereby releasing the drug relatively faster.

The drug-loading and the drug entrapment efficiencies in the formulated particles were enhanced by using an optimum amount of drug in preparatory steps ${ }^{27}$ and by using the proper solvent or solvent mixture. ${ }^{20}$ The drug entrapment was gradually increased with the decreasing size of nanoparticles. Our results showed that drug content was higher in drug-loaded polymeric nanoparticles without gold compared to nanoparticles with colloidal gold. No physical interaction, as assessed by FTIR, was found between drug and gold. Gold nanoparticles allow display of a large number of carbohydrates and other similar molecules with a high density and multifunctionality by the simultaneous incorporation of different ligands in a single gold cluster in a controlled manner. ${ }^{28}$ However, the lack of any distinct physicochemical interaction between the drug and the PLGA suggests that no such phenomenon took place. The presence of nanoscale-size gold particles within the polymeric nanoparticles containing drug results in less space available for the remaining drug compared to the nanoparticle containing drug without gold. Thus, the drug content was less with the polymeric nanoparticle containing gold. In addition, the presence of gold enhances the total weight of the nanoparticles, which in turn reduces the percentage of drug loading compared to the formulations without gold since the percentage of drug loading is 100 times the amount of drug present per unit quantity of nanoparticle. Thus, in spite of the presence of gold nanoparticles, which have an increased surface area to volume ratio, polymeric nanoparticles containing gold appeared to accommodate less drug molecules. In addition, drug content in the nanoparticles increased with the incorporation of more drug in the formulation. However, due to time constraints we could not determine the optimum amount of drug to achieve maximum drug loading.

In vitro drug release profiles of stavudine from PLGA nanoparticles showed that the cumulative percentage of drug release was about $70 \%$ of the drug content of the formulations in 63 days. The results support a steady and slow drug release from the experimental nanoparticles. In vitro release

Table 4 Nanoparticle (S4) uptake by macrophages

\begin{tabular}{lllll}
\hline $\begin{array}{l}\text { Serial } \\
\text { no }\end{array}$ & $\begin{array}{l}\text { Concentration of } \\
\text { nanoparticles in suspension } \\
\text { (ng/mL medium) }\end{array}$ & $\begin{array}{l}\text { Effective } \\
\text { concentration of drug } \\
\text { (ng/mL medium) }\end{array}$ & $\begin{array}{l}\text { Incubation } \\
\text { time } \\
\text { (hours) }\end{array}$ & $\begin{array}{l}\text { Degree of nanoparticles uptake by } \\
\text { macrophages (number of }+ \text { symbols } \\
\text { indicates the increasing degree) }\end{array}$ \\
\hline 1 & 300 & 17.28 & 4 & + \\
2 & 500 & 28.80 & 4 & ++ \\
\hline
\end{tabular}



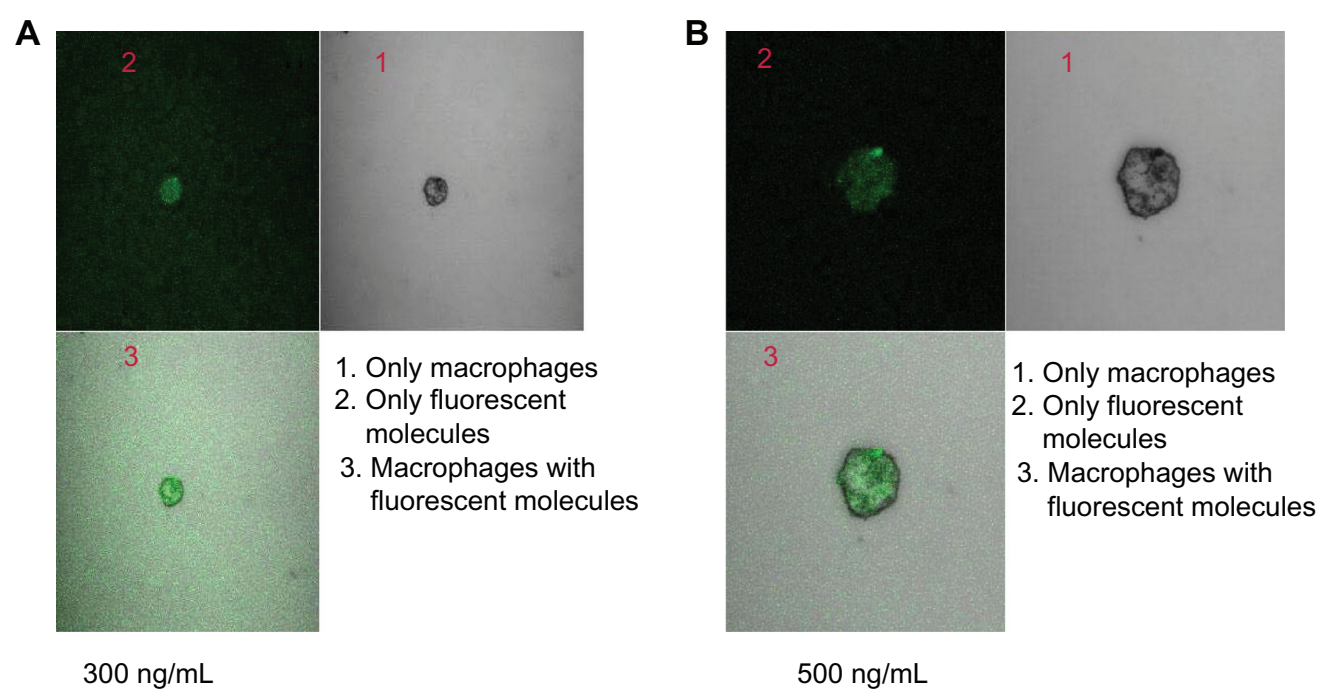

Figure 7 Confocal microscopic image of macrophages treated with (A) stavudine nanoparticles (300 ng/mL of medium) for 4 hours, and (B) stavudine nanoparticles (500 ng/mL of medium) for 4 hours.

data of stavudine from the nanoparticles were tested by the Higuchian kinetic model, which describes the release of drug from an insoluble matrix as dependent on the time based on Fickian diffusion. The release constant was calculated from the slope of the appropriate plots, and the regression coefficient was determined (Table 3 ). In vitro release of stavudine was best explained by Higuchi's equation as the plots showed the highest linearity followed by first-order kinetics. The corresponding plot for the Korsmeyer-Peppas equation indicated a good linearity, particularly for S3 and S4. The release exponent " $n$ " had values in the range of 0.45 and 0.89 , indicating that drug release was controlled by anomalous diffusion, ie, diffusion and erosion..$^{16-18}$ To confirm the erosion mechanism of drug from the nanoparticles, the Hopfenberg kinetic model ${ }^{17}$ was fitted to correlate the drug release from surface-eroding polymers as long as the surface area remains constant during the degradation process. The linearity range ( 0.914 to 0.985 ) was good, suggesting that the mechanism of drug release is controlled simultaneously by diffusion and erosion. ${ }^{16-18}$

Targeting of stavudine-loaded nanoparticles to macrophages has been assumed to more effectively kill HIV as the virus accumulates in macrophages. ${ }^{6}$ In addition, the nanoparticles were internalized by the macrophages in vitro, as assessed by FITC fluorescence intensity. Increasing the nanoparticulate concentration increased the fluorescent intensity, at least for a period of 4 hours.

\section{Conclusion}

Stavudine was released from the experimental formulations in a sustained manner over a prolonged period of time, and this would minimize the frequency of dosing interval. A faster drug-release pattern was observed for nanoparticles containing colloidal gold compared to those without gold. The presence of colloidal gold decreased drug loading in PLGA nanoparticles. Drug release patterns appeared to follow anomalous diffusion, and macrophages, an important target group of cells in early HIV infection, were found to internalize nanoparticles (S4), suggesting that dose-related toxicity would be less. Polymer-encapsulated colloidal gold nanoparticle uptake by macrophages provides support for the use of gold nanoparticles as diagnostic and therapeutic agents, but further studies are necessary to confirm this.

\section{Acknowledgments}

We are indebted to the Department of Biotechnology, Government of India (Grant no BCIL/NER-BPMC/2012/650) and the Indian Council of Medical Research (Grant no 45/20/2011/NAN/BMS) for partially funding the research work.

\section{Disclosure}

The authors report no conflicts of interest in this work.

\section{References}

1. Kreuter J. Evaluation of nanoparticles as drug delivery systems. I. Preparation method. Pharm Acta Helv. 1983;58(7):196-209.

2. Wang X, Wang Y, Chen Z, Shin DM. Advances of cancer therapy by nanotechnology. Cancer Res Tre. 2009;41(1):1-11.

3. Soppimath KS, Aminabhavi TM, Kulkarni AR, Rudzinski WE. Biodegradable polymeric nanoparticles as drug delivery devices. $J$ Controlled Release. 2001;70(1-2):1-20.

4. Sepkowitz KA. AIDS - the first 20 years. $N$ Engl J Med. 2001;344(23): 1764-1772. 
5. Weiss RA. How does HIV cause AIDS? Science. 1993;260(5112): 1273-1279.

6. Deneka M, Pelchen-Matthews A, Byland R, Ruiz-Mateos E, Marsh M. In macrophages, HIV-1 assembles into an intracellular plasma membrane domain containing the tetraspanins CD81, CD9, and CD53. J Cell Biol. 2007;177(2):329-341.

7. Storhoff JJ, Elghanian R, Mucic RC, Mirkin CA, Letsinger RL. One pot colorimetric differentiation of polynucleotides with single base imperfections using gold nanoparticle probes. J Am Chem Soc. 1998; 120(9):1959-1964.

8. Patra HK, Banerjee S, Chaudhuri U, Lahiri P, Dasgupta AK. Cell selective response to gold nanoparticles. Nanomedicine. 2007;3(2): 111-119.

9. Boisselier E, Astruc D. Gold nanoparticles in nanomedicine: preparations, imaging, diagnostics, therapies and toxicity. Chem Soc Rev. 2009; 38(6):1759-1782.

10. Mukherjee B, Patra B, Layek B, Mukherjee A. Sustained release of acyclovir from nano-liposomes and nano-niosomes. Int J Nanomedicine. 2007;2(2):213-225.

11. Bilensoy E, Gürkaynak O, Lale Doğanand A, Atilla Hıncal A. Safety and efficacy of amphiphilic ß-cyclodextrin nanoparticles for paclitaxel delivery. Int J Pharm. 2008;347(2):163-170.

12. Fu XD, Gao YL, Ping QN, Ren T. Preparation and in vivo evaluation of huperzine A-loaded PLGA microspheres. Arch Pharm Res. 2005;28(9): 1092-1096.

13. Mandal TK, Bostanian LA, Graves RA, Chapman SR. Poly(D,L-lactide-co-glycolide) encapsulated poly(vinyl alcohol) hydrogel as a drug delivery system. Pharm Res. 2002;19(11): 1713-1719.

14. Wang N, Wu XS, Li JK. A heterogeneously structured composite based on poly (lactic-co-glycolic acid) microspheres and poly (vinyl alcohol) hydrogel nanoparticles for long-term protein drug delivery. Pharm Res. 1999;16(9):1430-1435.

15. Arnáiz B, Martínez-Ávila O, Falcon-Perez JM, Penadés S. Cellular uptake of gold nanoparticles bearing HIV gp120 oligomannosides. Bioconjug Chem. 2012;23(4):814-825.

16. Pattnaik G, Sinha B, Mukherjee B, et al. Submicron-size biodegradable polymer-based didanosine particles for treating HIV at early stage: an in vitro study. J Microencapsul. 2012;29(7):666-676.
17. Dash S, Murthy PN, Nath L, Chowdhury P. Kinetic modeling on drug release from controlled drug delivery systems. Acta Pol Pharm. 2010; 67(3):217-223.

18. López-Gasco P, Iglesias I, Benedí J, Lozano R, Teijón JM, Blanco MD. Paclitaxel-loaded polyester nanoparticles prepared by spray-drying technology: in vitro bioactivity evaluation. J Microencapsul. 2011; 28(5):417-429.

19. Shan M, Klasse PJ, Banerjee K, et al. HIV-1 gp120 mannoses induce immunosuppressive responses from dendritic cells. PLoS Pathog. 2007; 3(11):e169.

20. Falcón-Pérez JM, Nazarian R, Sabatti C, Dell'Angelica EC. Distribution and dynamics of Lamp1-containing endocytic organelles in fibroblasts deficient in BLOC-3. J Cell Sci. 2005;118(Pt 22):5243-5255.

21. Freese C, Gibson MI, Klok HA, Unger RE, Kirkpatrick CJ. Size- and coating-dependent uptake of polymer-coated gold nanoparticles in primary human dermal microvascular endothelial cells. Biomacromolecules. 2012;13(5):1533-1543.

22. Silverstein RM, Webster FX. Spectrometric Identification of Organic Compounds. 6th ed. New York: John Wiley \& Sons; 1998:71-143.

23. Panyam J, Dali MM, Sahoo SK, et al. Polymer degradation and in vitro release of a model protein from poly (D,L-lactide-co-glycolide) nano- and microparticles. J Control Release. 2003;92(1-2):173-187.

24. Patil S, Sandberg A, Heckert E, Self W, Seal S. Protein adsorption and cellular uptake of cerium oxide nanoparticles as a function of zeta potential. Biomaterials. 2007;28(31):4600-4607.

25. Song C, Labhasetwar V, Cui X, Underwood T, Levy RJ. Arterial uptake of biodegradable nanoparticles for intravascular local drug delivery: results with an acute dog model. J Control Release. 1998;54(2):201-211.

26. Mocanu A, Cernica I, Tomoaia G, Bobos L-D, Horovitz O, Tomoaia-Cotisel M. Self-assembly characteristics of gold nanoparticles in the presence of cysteine. Colloids Surf A Physicochem Eng Asp. 2009; 338(1-3):93-101.

27. Sehra S, Dhake AS. Formulation and evaluation of sustained release microspheres of poly-lactide-co-glycolide containing tamoxifen citrate. J Microencapsul. 2005;22(5):521-528.

28. Ojeda R, de Paz JL, Barrientos AG, Martin-Lomas M, Penadés S. Preparation of multifunctional glyconanoparticles as a platform for potential carbohydrate-based anticancer vaccines. Carbohydr Res. 2007;342(3-4):448-459
International Journal of Nanomedicine

\section{Publish your work in this journal}

The International Journal of Nanomedicine is an international, peerreviewed journal focusing on the application of nanotechnology in diagnostics, therapeutics, and drug delivery systems throughou the biomedical field. This journal is indexed on PubMed Central,

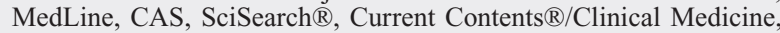

\section{Dovepress}

Journal Citation Reports/Science Edition, EMBase, Scopus and the Elsevier Bibliographic databases. The manuscript management system is completely online and includes a very quick and fair peer-review system, which is all easy to use. Visit http://www.dovepress.com/ testimonials.php to read real quotes from published authors. 